\title{
Analysis of Trans-Neptunian and Centaur colours: continuous trend or grouping?
}

\author{
M. A. Barucci ${ }^{1}$, M. Fulchignoni ${ }^{1,2}$, M. Birlan ${ }^{1}$, A. Doressoundiram ${ }^{1}$, J. Romon $^{1}$, and H. Boehnhardt ${ }^{3}$ \\ 1 Observatoire de Paris, DESPA, 5 place Jules Janssen, 92190 Meudon, France \\ 2 Université de Paris VII, Paris, France \\ 3 ESO, Alonso de Cordova 3107, Santiago de Chile, Chile
}

Received 4 July 2000 / Accepted 2 April 2001

\begin{abstract}
We report the results of the first statistical analysis of colours $(B-V, V-R, V-I$, and $V-J)$ of the Trans-Neptunian and Centaur populations. Using the same statistical techniques applied to define the current asteroid taxonomy, we find a continuous spread of the objects between neutral colour to very red. Pushing further the analysis, the TNOs may be split into four groups. The differences in colour content are interpreted as a consequence of the TNOs evolution (i.e. collisional history, space weathering, ...)
\end{abstract}

Key words. trans-Neptunian object - taxonomy - photometry - statistics

\section{Introduction}

The outer regions of the Solar System have recently been found to be densely populated by Trans Neptunian Objects (TNOs) forming the so-called Edgeworth Kuiper belt. The number of known TNOs is increasing continuously and since 1992 (Jewitt \& Luu 1993) more than four hundred TNO discoveries have been reported in IAU circulars (Marsden 2001). The study of these objects has rapidly evolved in the past few years, especially from dynamical and theoretical points of view. Perturbations of TNOs due to the gravitational influence of the outer planets can inject some of these bodies into giant-planet crossing orbits characteristic of the Centaurs (Stern 1996; Duncan et al. 1995; Stern \& Campins 1996). The Centaurs seem to be evolved from TNOs; this is further supported by the few physical studies of their surface, which indicate similar surface nature. The studies of physical and chemical properties of TNO population are still limited by the difficulties in observing these faint and elusive objects. Most of the known TNOs have a diameter between $100 \mathrm{~km}$ and $1200 \mathrm{~km}$. In this range, up to $10^{5}$ objects are estimated to orbit in a zone between 30 and $50 \mathrm{AU}$ from the Sun (Jewitt 2000). Careful observational and image processing procedures have been used to obtain reliable photometric data of TNOs. This is a very challenging task, and $B-V, V-R$, and $V-I$ colours are available for about 40 objects (Luu \& Jewitt 1996; Davies et al. 1998; Jewitt \& Luu 1998; Tegler \& Romanishin 1998; Barucci et al. 1999; Barucci et al. 2000; Tegler \& Romanishin 2000;

Send offprint requests to: M. A. Barucci,

e-mail: antonella.barucci@obspm.fr
Doressoundiram et al. 2001) and only 22 out of them have $V-J$ colour determined (Davies et al. 1998; Jewitt \& Luu 1998; Davies et al. 2000; Boehnhardt et al. 2001).

Back to the sixties, $U-B$ and $B-V$ colours were available for about 40 asteroids and these data represented the only physico-chemical data base concerning the asteroids at that time. Wood \& Kuiper (1963), analysing the data set, found that the asteroid population was split in two distinct groups in the $U-B$ vs. $B-V$ plane. One group clustered close the colours of the Sun while the other one clustered around the colours of the Moon. The availability of more detailed data allows us to obtain a much more detailed picture of the asteroid population, which contains several different compositional classes of objects described in a widely accepted taxonomic scheme, obtained by different authors with different multivariate statistical methods (Barucci et al. 1987; Tholen \& Barucci 1989; Tedesco et al. 1989). The Wood and Kuiper groups can be considered as the "seeds" of the asteroid C (for carbonaceous), and S (for silicaceous) class respectively.

As far as the Kuiper Belt is concerned, Tegler \& Romanishin (1998) using the $B-V$ and $V-R$ colours of 13 objects ( 9 TNOs and 4 Centaurs) found a subdivision of those objects into two groups, consisting of objects having colours slightly redder than the colour of the Sun (corresponding to the $\mathrm{C}$ and $\mathrm{D}$ asteroid classes) and the other containing the reddest objects known in Solar System.

\section{Statistical analysis}

The availability of large telescopes for TNO research has resulted in an increase of the number of observed objects. 
Table 1. Colours of the analysed objects: the first 15 are TNOs, the last 7 are Centaurs

\begin{tabular}{cccccc}
\hline Objects & $B-V$ & $V-R$ & $V-I$ & $V-J$ & Ref. \\
\hline \hline Sun & 0.67 & 0.36 & 0.69 & 1.08 & 1,2 \\
\hline 1993 SC & $1.04 \pm 0.17$ & $0.65 \pm 0.09$ & $1.39 \pm 0.10$ & $2.27 \pm 0.08$ & $4,5,6,7$ \\
1994 TB & $1.03 \pm 0.18$ & $0.76 \pm 0.15$ & $1.41 \pm 0.15$ & $2.54 \pm 0.11$ & $3,4,5,6$ \\
1995 QY9 & $0.71 \pm 0.20$ & $0.47 \pm 0.12$ & $0.87 \pm 0.06$ & $2.01 \pm 0.13$ & $3,5,6$ \\
1996 TL66 & $0.69 \pm 0.08$ & $0.31 \pm 0.16$ & $0.71 \pm 0.16$ & $1.26 \pm 0.11$ & $3,4,6,7$ \\
1996 TO66 & $0.67 \pm 0.08$ & $0.37 \pm 0.08$ & $0.76 \pm 0.08$ & $0.86 \pm 0.14$ & $3,4,6,7,8$ \\
1996 TP66 & $1.04 \pm 0.19$ & $0.66 \pm 0.09$ & $1.23 \pm 0.14$ & $2.16 \pm 0.07$ & $3,4,6,7$ \\
1996 TS66 & $0.98 \pm 0.13$ & $0.60 \pm 0.17$ & $1.27 \pm 0.17$ & $1.87 \pm 0.11$ & $4,6,7$ \\
1997 CS29 & $1.07 \pm 0.07$ & $0.64 \pm 0.04$ & $1.19 \pm 0.04$ & $2.06 \pm 0.03$ & $4,6,9$ \\
1997 CQ29 & $0.99 \pm 0.12$ & $0.68 \pm 0.06$ & $1.30 \pm 0.09$ & $1.84 \pm 0.37$ & 6,9 \\
1998 SN165 & $0.82 \pm 0.08$ & $0.40 \pm 0.08$ & $0.84 \pm 0.07$ & $2.18 \pm 0.12$ & 10,11 \\
1999 TC36 & $0.96 \pm 0.04$ & $0.70 \pm 0.04$ & $1.28 \pm 0.08$ & $2.24 \pm 0.04$ & 12 \\
1998 TF35 & $1.02 \pm 0.11$ & $0.71 \pm 0.06$ & $1.28 \pm 0.15$ & $2.30 \pm 0.13$ & 12 \\
1998 VG44 & $0.93 \pm 0.05$ & $0.61 \pm 0.04$ & $1.38 \pm 0.08$ & $2.31 \pm 0.08$ & 12 \\
1998 WH24 & $0.93 \pm 0.06$ & $0.59 \pm 0.04$ & $1.26 \pm 0.06$ & $1.83 \pm 0.03$ & $6,9,16$ \\
1998 XY95 & $0.93 \pm 0.23$ & $0.65 \pm 0.14$ & $1.42 \pm 0.30$ & $2.35 \pm 0.24$ & 12 \\
2060 Chiron & $0.70 \pm 0.02$ & $0.37 \pm 0.03$ & $0.72 \pm 0.04$ & $1.20 \pm 0.30$ & 13,14 \\
5145 Pholus & $1.27 \pm 0.10$ & $0.78 \pm 0.03$ & $1.55 \pm 0.02$ & $2.59 \pm 0.02$ & 13,14 \\
7066 Nessus & $1.09 \pm 0.04$ & $0.79 \pm 0.04$ & $1.50 \pm 0.10$ & $2.29 \pm 0.04$ & 13,14 \\
8405 Asbolus & $0.75 \pm 0.04$ & $0.44 \pm 0.03$ & $0.96 \pm 0.03$ & $1.65 \pm 0.02$ & 13,14 \\
10199 Chariklo & $0.77 \pm 0.05$ & $0.47 \pm 0.02$ & $1.01 \pm 0.02$ & $1.74 \pm 0.02$ & 13,14 \\
10370 Hylonome & $0.70 \pm 0.08$ & $0.43 \pm 0.10$ & $0.87 \pm 0.10$ & $1.31 \pm 0.10$ & $4,5,13,15$ \\
1998 SG35 & $0.89 \pm 0.11$ & $0.43 \pm 0.08$ & $1.02 \pm 0.08$ & $1.35 \pm 0.19$ & 10,11 \\
\hline
\end{tabular}

1, Hardorp (1980); 2, Hartmann et al. (1982); 3, Barucci et al. (1999); 4, Tegler \& Romanishin (1998); 5, Luu \& Jewitt (1996); 6, Davies et al. (2000); 7, Jewitt \& Luu (1998); 8, Hainaut et al. (2000); 9, Barucci et al. (2000); 10, Doressoundiram et al. (2001); 11, Doressoundiram (private comunication); 12, Boehnhardt et al. (2001); 13, Davies et al. (1998); 14, Davies (2000); 15, Green et al. (1997); 16, Tegler \& Romanishin (2001).

Observations through $B, V, R, I$, and $J$ filters have been obtained by various authors for several objects, but the complete set is available for 22 objects (15 TNOs and 7 Centaurs). We analysed the sample of the TNO population constituted by these 22 objects described by the four colour variables $B-V, V-R, V-I$, and $V-J$ with the goal of investigating its structure. The data used are listed in Table 1. When multiple observations of an object were available in the literature, we adopted the average value for the colour and we took for the uncertainty the maximum of either the dispersion of the values or formal error reported. The observation procedure used by most of the observers consist in intercalating consecutive observations through other filters with multiple observations through the same filter to allow interpolation (e.g. $V-B-V-R-V-I-V-J-V)$. A luminosity variation due to the rotation would affect in similar way the consecutive observations in the different filters, so the colour determination would not be strongly affected by rotational effect. This method together with the use of the average of multiple observations allow us to reduce the effects of possible luminosity variation. We carried out our analysis using both of the techniques used in obtaining the current asteroid taxonomy (Barucci et al. 1987; Tholen \& Barucci 1989): a) the Principal Component Analysis (PCA) (Reyment \& Joreskog 1993) and b) the G-mode analysis (Coradini et al. 1977; Fulchignoni et al. 2000).
For details on these statistical techniques, the reader is remainded to the quoted literature.

The principal components are the eigenvectors of the variance-covariance matrix of the colours. The principal components are linear combinations of the original variables where the coefficients (eigenvectors) reflect the relative importance of a variable within a principal component. The sum of the eigenvalues of this matrix is equal to its trace, i.e. it accounts for the total variance of the sample. Each eigenvalue reflects the percentage of the total variance contributed by each principal component. In Table 2 the results of this analysis are reported. The first eigenvector accounts for most of the variance of the sample (91.14\%), and heavily weights the contribution of $V-J$ (48\%). The second eigenvector adds only $7.33 \%$ of the total variance and is weighted mainly by both $V-I(35 \%)$ and $V-J(29 \%)$. Therefore, we infer that first principal component PC1 and second principal component PC2 measure the degree of reddening of the TNOs' surfaces, which is the main distinctive character of this population members. The last eigenvectors contribute negligibly to the total variance, so the $\mathrm{PC} 1$ vs. PC2 plane contains practically all the information on the variance of variables characterising the sample. In Fig. 1 the predominance of PC1 in representing most of the sample variance is reflected: the objects spread all along this axis with scores spanning from 1.2 to 3.3 , while the $\mathrm{PC} 2$ scores range 


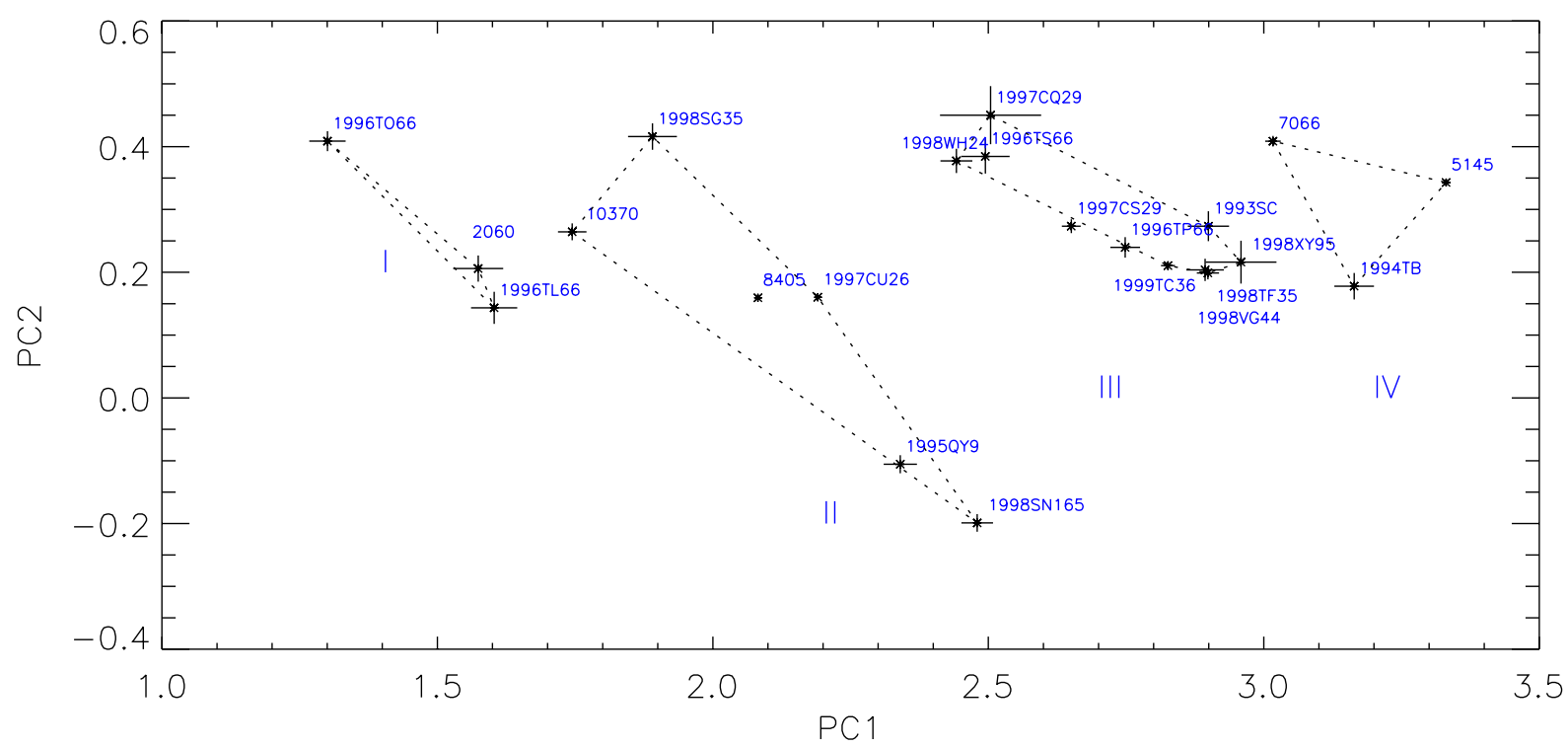

Fig. 1. TNO and Centaur designation for each object in the PC1 vs. PC2 plot are reported. The predominance of PC1 is evident. The spreading of the analysed sample is a clear indication of a continuous trend from neutral (lower PC1 scores) to very red (higher PC1 scores). The dotted lines include the objects belonging to each group found by the G-mode analysis. The error bars of PC1 and PC2 have been obtained propagating the errors of the colours

only between -0.2 and 0.5 . We interpreted this behaviour as a net indication of a continuous trend from neutral (lower PC1 scores) to very red (higher PC1 scores) surface colours. By examining the PC1 and PC2 components in more details, a finer structure of the sample seems to overlap the continuous trend (see Fig. 1).

In order to check the existence of this finer structure we analysed the same sample with the G-mode multivariate statistics (Coradini et al. 1977). This method allows us to analyse a sample of $\mathrm{N}$ objects described by $\mathrm{M}$ variables with the aim of separating, on the basis of an appurtenance test, the $\mathrm{N}$ objects in groups which are homogeneous in terms of the variables content. The method also gives an indication of the relative importance of the variables in separating the groups. An estimation of the statistical distance of the groups is also obtained by computing the total variance of all of the variables of one group with respect to the means of all the others groups, which can be interpreted in terms of possible processes affecting (or linking) the different groups.

The G-mode analysis separates the 22 objects of our sample in four groups. In Fig. 2 the average broad band colours obtained for each group are presented as reflectance spectra, normalised to the Sun and to $V$ colour. The colours are transformed in reflectance values by $R_{c_{\lambda}}=$ $10^{ \pm 0.4\left(c_{\lambda}-c_{\lambda_{0}}\right)}$ where $c_{\lambda}$ and $c_{\lambda_{0}}$ are the $\lambda-V$ colours of the object and of the Sun respectively. Group I contains the objects having a neutral reflectance spectrum, group IV contains the reddest objects in the Solar System. Groups II and III have transitional character between group I and group IV respectively, as far as $B-V, V-R$ are concerned, but are clearly distinguished by $V-I$ and $V-J$. This splitting has been obtained at a confidence level of $99 \%$. The importance of variables in obtaining the four groups is $42 \%$ for the $V-I$ colour, $26 \%$ for $V-J$, $20 \%$ for $V-R$ and $12 \%$ for $B-V$. The $V-I$ variable discriminates each group from all the others at an high significance level, the $V-J$ variable plays the same role at a lower level as well as $V-R$, while $B-V$ contribute weakly in separating the groups.

The general results of the G-mode analysis are i) within the sample, four groups of objects can be identified; ii) each group is characterised by a quite homogeneous set of variables; iii) the most important contribution in discriminating the groups comes from the variable $V-I$; iv) the statistical distances between the groups show that group I and group IV exhibit the larger differences, while groups II and III are closer because of their common intermediate behaviour, and they span between group I and group IV; v) these groups define the finer subdivision overlapping the continuous trend found in the PC1 vs. PC2 plot. Finally, has to be outlined that the asteroid taxonomy has been obtained by the G-mode (Barucci et al. 1987 ) at a lower significance lever ( 93\%): it may imply that the TNO's grouping could have as much relevance as the asteroid classification.

\section{Conclusion and discussion}

The results obtained by the two statistical methods used are fully compatible. The quasi-continuous spreading of the objects between two end members (those with neutral spectra and those with the reddest known spectra) results from the weight $(91.14 \%)$ of the first principal component. Both PCA and G-mode analysis show the same finer structure of the sample. This grouping is distinguished by their content of $V-I$ and $V-J$, while is quite marginal for $V-R$ and $B-V$. Due to the faintness of TNOs, 
observations are very difficult and consequently errors are quite important and are reflected on the colour determination. Changing the value of a colour of a single object can make it to change group. However the important point is not the belonging of one single object to one group or another, but the existence of subdivisions. The main results of our analysis is that $I$ and $J$ observations are critical in discriminating groups and for this reason we suggest to observe TNOs up to the infrared ( $I, J$ filters).

Our results exclude the claimed bimodality of the TNO population (Tegler \& Romanishin 1998) and confirm quantitatively by statistical analysis the interpretation done by previous authors regarding the evolution of TNO population. In fact the quasi-continuous colour variation of the TNOs can be a consequence of collisions at all scales and of space weathering (Luu \& Jewitt 1996; Davis \& Farinella 1997). High energy charged particles (cosmic and solar) bombardment induce a deposition of some of the organic compounds contained in the icy bodies of the TNOs, which forms a coating of dark materials characterised by a very red spectrum. Micrometeoroid or meteoroid impacts can locally remove the coating, exposing patches of the underlying surface (whose extension depends on the energy of the projectile) and lowering at different level the influence of the organic coating in reddening the spectra. Mutual collisions can have more dramatic effects in rejuvenating the TNO surfaces (Durda \& Stern 2000; Davis \& Farinella 1997): large impacts resurface the objects leading to neutral or lightly red spectra, as shown by the few known cometary nuclei. All the TNOs experience collisions (projectile $D>1 \mathrm{~km}$ ) at rate of $10^{-6}-10^{-8}$ per year (Davis \& Farinella 1997 ), so the population exhibits a continuous range of surface conditions, from the freshly exposed original neutral icy surfaces, to the heavily bombarded by charged particles, coated old red surfaces. The groups which appear to overlap this continuous trend when a finer structure is extracted can be interpreted as the superposition of some peculiar processes over the collisional evolution described. The available data are insufficient to allow us to interpret the meaning of the grouping. More observations are needed. As an example we note that for two of the objects belonging to group I, the presence of some activity has been claimed: coma outgassing has been detected (Meech \& Belton 1989) for 2060 Chiron and outbursts have been hypothesised (Hainaut et al. 2000) for $1996 \mathrm{TO}_{66}$. This kind of activity could change the reflectance properties of these objects due to the fact that the sunlight is reflected by the gaseous and dusty cloud surrounding these bodies. The possible resurfacing of the body by fresh debris could as well be the source of the similarity in the colour behaviour of group I objects.

The present analysis is based on a very small data set, so the four groups found may be not representative. Our results show that the TNO population characters are much more complex than those resulting from the previous analysis based on only marginally discriminating variables and are not bimodal. We have started two large observation

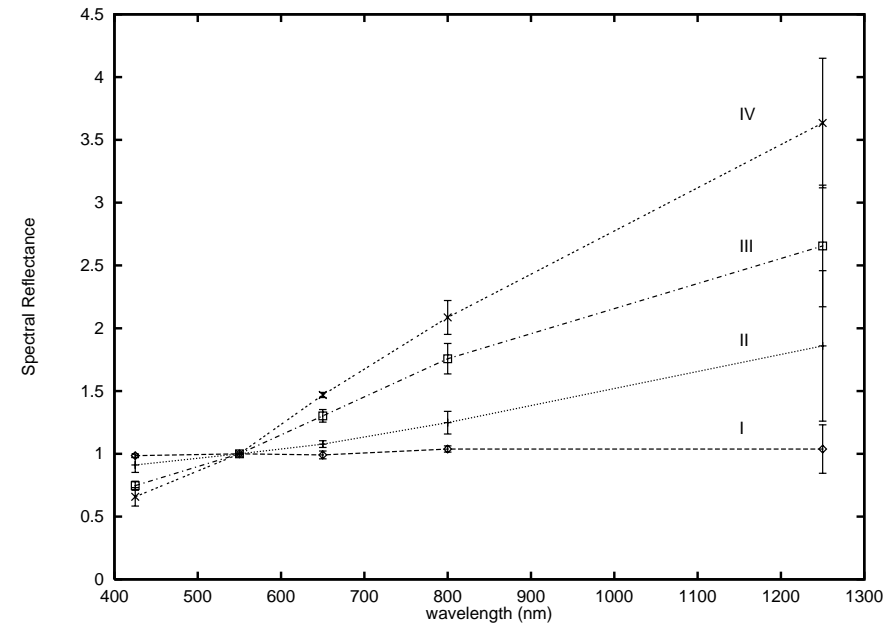

Fig. 2. The average broad band reflectance spectra normalised to $V$ band (centered at $550 \mathrm{~nm}$ ) are reported for each group found by G-mode analysis. The error bar is the standard deviation of the reflectance mean values within each group. The four groups spread continuously from neutral spectrum (group I) to very red one (group IV) containing the reddest objects of the solar system

Table 2. Eigenvectors, eigenvalues and $\%$ of total variance contributed by each eigenvalue

\begin{tabular}{ccccc}
\hline Variable & 1 & 2 & 3 & 4 \\
\hline \hline$B-V$ & 0.251 & 0.386 & -0.859 & 0.223 \\
$V-R$ & 0.238 & 0.314 & -0.027 & -0.918 \\
$V-I$ & 0.431 & 0.669 & 0.511 & 0.326 \\
$V-J$ & 0.833 & -0.552 & 0.002 & 0.028 \\
& & & & \\
Eigenvalues & 0.313 & 0.025 & 0.004 & 0.002 \\
\% of total variance & 91.141 & 7.331 & 1.066 & 0.463 \\
\hline
\end{tabular}

programmes, at VLT (ESO, Chile) and CFHT (Hawaii) to investigate the nature of these objects and to obtain a high quality homogeneous colour $(B-V, V-R, V-I$, $V-J, V-H$, and $V-K)$ set for a large sample of TNOs.

Acknowledgements. We thank A. W. Harris (JPL) for helpful discussions.

\section{References}

Barucci, M. A., Capria, M. T., Coradini, A., \& Fulchignoni, M. 1987, Icarus, 72, 304

Barucci, M. A., Doressoundiram, A., Fulchignoni, M., et al. 1999, Icarus, 142,476

Barucci, M. A., Romon, J., Doressoundiram, A., et al. 2000, AJ, 120, 496

Boehnhardt, H., Tozzi, G. P., Birkle, K., et al. 2001, A\&A, submitted

Coradini, A., Fulchignoni, M., Fanucci, O., et al. 1977, Comput. Geosci., 3, 85

Davies, J. K. 2000, in Minor Bodies of the Outer Solar System, ed. A. Fitzsimmons, D. Jewitt, \& R. M. West, ESO Astrophysics Symposia (Springer-Verlag Berlin Heidelberg) 9 
Davies, J. K., Green, S., McBride, N., et al. 2000, Icarus, 146, 253

Davies, J. K., McBride, N., Ellison, S. L., et al. 1998, Icarus, 134,213

Davis, D. R., \& Farinella, P. 1997, Icarus, 125, 50

Doressoundiram, A., Barucci, M. A., Romon, J., et al. 2001, Icarus, submitted

Duncan, M., Levison, H., \& Budd, S. 1995, AJ, 110, 3073

Durda, D. D., \& Stern, S. A. 2000, Icarus, 145, 220

Fulchignoni, M., Birlan, M., \& Barucci, M. A. 2000, Icarus, 146,204

Green, S. F., McBride, N., O'Cealleagh, D., et al. 1997, MNRAS, 290, 186

Hainaut, O. R., Dalahodde, C. E., Boehnhardt, H., et al. 2000, A\&A, 356, 1076

Hardorp, J. 1980, A\&A, 91, 221

Hartmann, W. K., Cruishank, D. P., \& Degewij, J. 1982, Icarus, 52,377

Jewitt, D. C. 2000, in Minor Bodies of the Outer Solar System, ed. A. Fitzsimmons, D. Jewitt, \& R. M. West, ESO Astrophysics Symposia (Springer-Verlag Berlin Heidelberg) 1
Jewitt, D. C., \& Luu, J. X. 1993, Nature, 362, 730

Jewitt, D., \& Luu, J. X. 1998, AJ, 115, 1667

Luu, J. X., \& Jewitt, D. 1996, AJ, 112, 2310

Marsden, B. 2001, http://cfa-www.harvard.edu/cfa/ps/ lists/TNOs.html

Meech, K. J., \& Belton, M. J. S. 1989, IAU Circ., 4770

Reyment, R., \& Joreskog, K. G. 1993, Applied Factor Analysis in natural Sciences (Cambridge Univ. Press)

Stern, S. A. 1996, in Completing the inventory of the Solar System, ed. T. W. Retting, \& J. M. Hahn, ASP Conf. Ser., 107, 209

Stern, S. A., \& Campins, H. 1996, Nature, 382, 507

Tedesco, E. F., Williams, J. G., Matson, D. L., et al. 1989, AJ, 97, 580

Tegler, S. C., \& Romanishin, W. 1998, Nature, 392, 49

Tegler, S. C., \& Romanishin, W. 2000, Nature, 407, 979

Tholen, D. J., \& Barucci, M. A. 1989, in Asteroid II, ed. R. P. Binzel, \& T. Gehrels (Univ. of Arizona Press), 298

Wood, H. J., \& Kuiper, G. P. 1963, AJ, 137, 1279 